\title{
Design and Implementation of the Digital Controller for Boost Converter based on FPGA
}

\author{
Bo LI, Shuibao GUO, Xuefang LIN-SHI, Bruno ALLARD, Senior Member, IEEE, \\ Lyon University, Ampere Laboratory, INSA Lyon, CNRS UMR 5005, Villeurbanne, 69621, France \\ bo.li2, shuibao.guo, xuefang.shi, bruno.allard@insa-lyon.fr
}

\begin{abstract}
Taking advantage of FPGA's attractive features, this paper presents an improved digital pulse-width-modulator (DPWM) based sliding-mode controller (SMC) for boost converter that effectively alleviates the quantization effects. The dithering Multi-stAge-noise-SHaping (MASH) DPWM is introduced exhibiting a better idle tone suppression effect that achieves relatively higher effective number of bit (ENOB). The Linear Feedback Shift Register (LFSR) replaces the cumbersome pseudo-random generator as the dither generation module that is proven to be more effective. The SMC with the proper sliding coefficients aiming at a better dynamic response than the traditional PID controller cooperates with our proposed DPWM. Two individual boards, an analog-to-digital converter (ADC) and a boost converter, connecting to a Virtex-II FPGA platform compose a close-loop test environment. Experimental results verify the switching-mode-power-supply (SMPS) closeloop operation at $1 \mathrm{MHz}$ switching frequency with an 11-bit effective DPWM resolution.
\end{abstract}

\section{INTRODUCTION}

Owing to the development of modern integrated circuit technology especially the field-programmable gate array (FPGA), digital controller for switching-mode-power-supply (SMPS) offers many advantages compared to the readily available analog counterparts which still dominate the current portabledevice market. Meanwhile integration leads to very high switching frequency. Digital controllers are seldom proven above $10 \mathrm{MHz}$ of switching frequency. Electromagnetic interference (EMI) issues also lead to fixed frequency converters. The challenge is to accommodate both constraints and satisfactory dynamic performances but with a reasonable efficiency. Therefore many solutions are proposed to solve the problems of the controller. Figure 1 shows the three main parts of the digital feedback loop for a boost converter. Analog-to-digital converter (ADC) acquires and scales the output of the boost converter and provides digital signal to the controller. Benefiting from the digital approach, the control law block can implement advanced algorithms to reach better control performance. Digital pulse-width-modulator (DPWM) is suitable to convert the control signal to a variable duty cycle signals which drive the switches in the power stage.

A DC-DC converter is a kind of variable structure system (VSS) due to the intrinsic switching nature [1], [2]. Meanwhile, a sliding-mode controller (SMC) is a nonlinear controller which offers an alternative to implement a control action which exploits the inherent variable structure nature of the DC-DC converter as apposed to the analog control implementation. In order to address the requirement of fixed

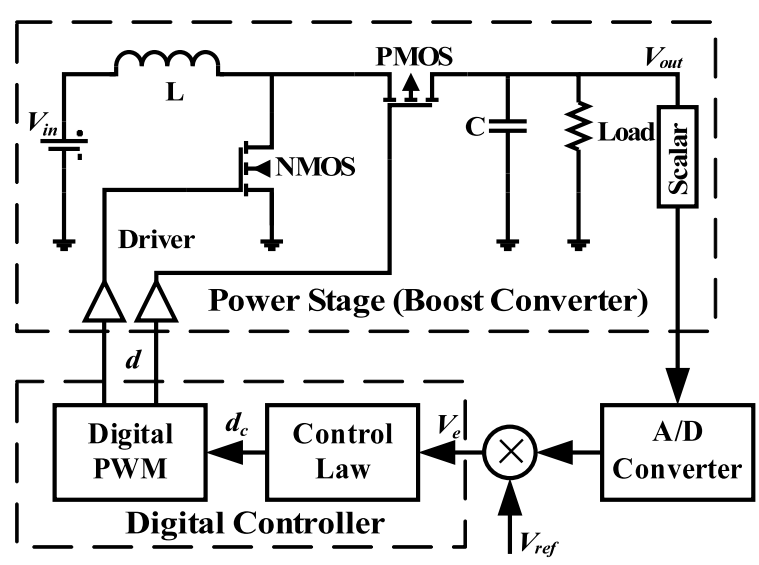

Fig. 1. Circuit structure of digitally controlled boost converter
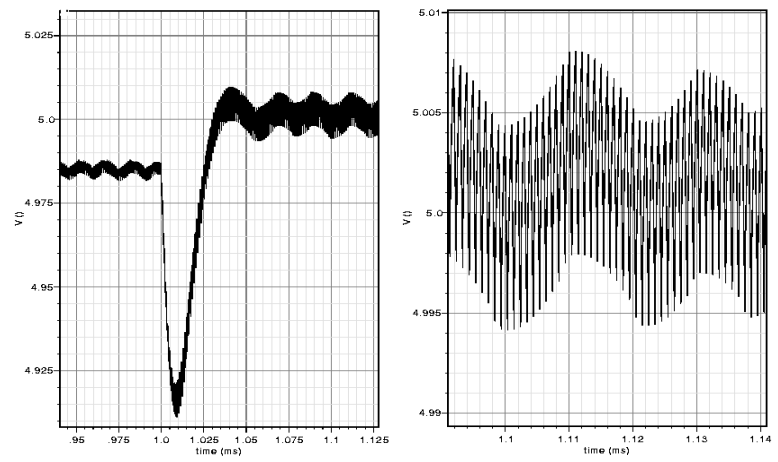

Fig. 2. Simulated LCO output of the boost converter with 10-bit ADC and 8-bit DPWM

and controlled switching frequency, a PWM-based SMC is derived from a Hysteresis Modulator (HM) based SMC [3], [4] and [5]. It has been also proposed in [6] and [7], where the switching frequency is in the range of hundreds of $\mathrm{kHz}$. Unfortunately, most of the published papers only verify SMC in analog approach that limits the bandwidth, as well as reduces the flexibility of implementation [8], [6]. The abovementioned SMC is the PWM-based SMC that can implement in FPGA to generate a control signal employing the concept of equivalent control [9]. The SMC is relatively fast to achieve desired specification and is robust with respect to matched internal and external disturbances.

Due to the quantization effect that both exists in ADC 
and DPWM, an limit-cycle oscillation (LCO) may occur that imposes restrictions on not only the compensator but also DPWM [10], [11]. As shown in figure 2, obvious LCO may be introduced if the quantization step of the DPWM is too coarse. According to the static and dynamic conditions proposed by [10], there is no doubt that a high-resolution DPWM is a necessary condition to avoid the LCO. Relying on the excellent linearity, the advantages of the counter-comparator DPWM is obvious in the time-domain; nevertheless, for a $n$-bit DPWM dedicating to a boost converter of which the switching frequency is $f_{s}$, an external clock must reach $2^{n} \cdot f_{s}$ theoretically that is rarely achievable with the current integrated circuit technology or at the risk of large power consumption as well as the necessitate for an elaborate clock tree. In order to overcome the above-mentioned problems and achieve enough precise voltage, a delay-line DPWM [12], a hybrid delay-line [13], a segment delay-line DPWM [14] and a ring-MUX DPWM [15] are proposed to obtain a delay time by adopting a series of tight logic cells. However these approaches suffer from manufacturing variability and occupy large chip area. Based on the "step calculation" principle, the dithering DPWM is proposed to achieve a high-resolution in a software manner without increasing the chip area and power consumption [11], nevertheless, the ripple in the output voltage is aggravated, so the effective number of bit (ENOB) is limited.

Delta-sigma $(\Delta-\Sigma)$ modulator (MOD) is a well-known oversampling structure that samples at a over-sampling rate (OSR) to realize noise-shaping effect [16]. Referring to the power stage of boost converter as the post-filter in digital-to-analog converter (DAC), DPWM can also adopts the over-sampling technique to pre-process the most-significant-bit (MSB) $m$-bit of the duty cycle command $d_{c}$, in other words, the required external clock frequency can reduce to $2^{n-m} \cdot f_{s}$, where $n$ is the length of $d_{c}$. However the slow convergence due to a low frequency periodic behavior in a first-order $\Delta-\Sigma$ DPWM, which is known as idle tone effects, produces a lowfrequency noise in the power stage. Meanwhile higher order implementations may bring potential stability problems [16], [17]. The Multi-stAge-noise-SHaping (MASH) type [18] is an effective trade-off between the first-order and higher-order $\Delta-\Sigma$ DPWM, while low-frequency tone still exists. In this paper, an insight into the idle tone effects for $\Delta-\Sigma$ DPWM, particularly for MASH $\Delta-\Sigma$ DPWM, is presented. Based on the analyses of two solutions that can suppress the idle tone, higher order modulator or dither[16], an efficient dither 1-1 MASH structure is employed.

The paper is organized as follows. Section II gives details on the implementation method of the SMC for the boost converter. Section III analyzes the idle tone suppression technique and describes the proposed DPWM. All proposals are verified experimentally in a FPGA based platform, and the results are discussed in section IV.

\section{PWM-BASED SMC FOR BOOST CONVERTER}

The small signal and average model for boost converter can be express as

$$
\begin{aligned}
& \dot{I}_{L}=-\frac{1-D}{L} V_{o}+\frac{1}{L} V_{i n} \\
& \dot{V}_{o}=\frac{1-D}{C} I_{L}-\frac{1}{R C} V_{o}
\end{aligned}
$$

where the two state variables $I_{L}$ and $V_{o}$ stand for the inductor current and output voltage; $C, R$ and $L$ are the capacitance, load resistance and inductance, respectively; $V_{i n}$ and $D$ are the input voltage and duty cycle to the boost converter.

The sliding surface can be introduced as [19], [4] and [20]

$$
S(t)=k_{1} \cdot e+k_{2} \cdot \dot{e}+k_{3} \cdot \int e d t
$$

where $k_{1}, k_{2}$ and $k_{3}$ represent the control parameters, $e$ is $\left(V_{\text {ref }}-V_{o}\right) . V_{\text {ref }}$ and $V_{o}$ are the reference and output voltage respectively. According to the equivalent control concept, the tracking error will be converged to zero if the invariance conditions are verified. In other words, the system is definitely trapped on the sliding surface leading to

$$
S(t)=\dot{S}(t)=0
$$

For power converters, the duty cycle $D$ can be deduced from the equivalent control function, provided that the switching frequency is relatively high [6], thus the converter is controlled here by varying the duty cycle and keeping the switching frequency constant, that could be expressed as

$$
\begin{array}{r}
D=\frac{\left(V_{o}-V_{i n}\right)}{V_{o}}+\frac{k_{3}}{k_{2}} \cdot L \cdot C \cdot \frac{\left(V_{r e f}-V_{o}\right)}{V_{o}} \\
-\left(\frac{k_{1}}{k_{2}}-\frac{1}{R \cdot C}\right) \cdot \frac{L \cdot C}{V_{o}} \cdot \frac{d V_{o}}{d t}
\end{array}
$$

The Lyapunov function can be chosen to determine the motion range [21]. Using the Routh's stability criterion, the stability conditions can be satisfied if all the parameters $k_{1}, k_{2}$ and $k_{3}$ have the same sign. The convergence dynamics can be chosen as a standard second-order system form:

$$
s^{2}+2 \xi \omega_{n} s+\omega_{n}^{2}=0
$$

where the damping ratio $\xi$ and undamped natural frequency $\omega_{n}$ are respectively:

$$
\omega_{n}=\sqrt{k_{3} / k_{2}}, \quad \xi=k_{1} / 2 \sqrt{k_{3} k_{2}}
$$

So the sliding parameters can be obtained by

$$
\begin{aligned}
& \frac{k_{3}}{k_{2}}=4 \pi^{2} f_{n}^{2} \\
& \frac{k_{1}}{k_{2}}=4 \pi \xi f_{n}
\end{aligned}
$$

where $f_{n}$ is the bandwidth of the controller's response. According to the simulation results, we set $\xi$ equals 0.75 , and $f_{n}=\frac{f_{s}}{60}$ in order to achieve better dynamic response performance. 


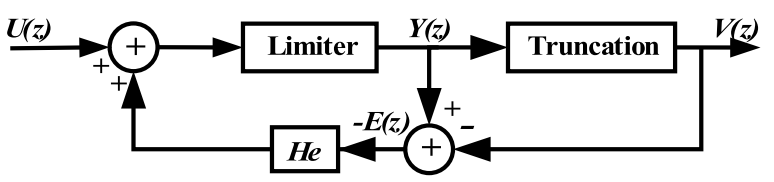

Fig. 3. Error feedback structure for DPWM

\section{THE DiTHER 1-1 MASH $\Delta-\Sigma$ DPWM}

To implement the proposed SMC in digital approach, a digital-PWM must be introduced. Owing to the inherent truncation configuration, the error feedback structure [16] turns out to be an efficient model for $\Delta-\Sigma$ DPWM as shown in figure 3 . The corresponding transfer function is

$$
V(z)=U(z)+[1-H e(z)] \cdot E(z)
$$

The first-order $\Delta-\Sigma$ modulation (MOD1) and the secondorder $\Delta-\Sigma$ modulation (MOD2) can be implemented by setting $H e(z)=z^{-1}$ and $H e(z)=z^{-1} \cdot\left(2-z^{-1}\right)$ separately [22], [17]. Taking MOD2 as an example, the signal transfer function $(S T F)$ and the noise transfer function $(N T F)$ are given by

$$
\begin{gathered}
\operatorname{STF}(z)=1 \\
\operatorname{NTF}(z)=\left(1-Z^{-1}\right)^{2}
\end{gathered}
$$

Therefore MOD2 can reach infinite gain at zero frequency as well as lead to the complete elimination of DC quantization errors, so

$$
\left.V(z)\right|_{z=1}=U(z)
$$

Although the noise-shaping can reduce the high frequency requirement for the core DPWM to some extent, the consequent idle tone introduces the in-band noise that only associates with DC input for a certain $\Delta-\Sigma$ DPWM. The power stage in SMPS works as the post analog low-pass filter in $\Delta$ $\Sigma$ DAC to suppress most of the out-of-band noise power in which the corner frequency $f_{o}=1 / 2 \pi \sqrt{L C}$ [23] must be sufficiently lower than the switching frequency and even than the frequency of the idle tone.

Higher-order modulator and dither are proven to be two feasible solutions in ADC to suppress the idle tone effects. The same method is compatible with DPWM. Many papers concentrated on the higher-order approach in which [17] exhibits a MOD2 adopting above-mentioned error feedback structure. An increasingly popular structure, MASH MOD, can partially solve the stability problems associated with highermodulators as shown in [18]. In figure 4, a MASH $\Delta-\Sigma$ DPWM is presented in which both stage contains a first-order internal loop, resulting in a global second-order noise-shaping effect, but preserving the robust stability as the first-order loop. In order to have the same noise-shaping effect as MOD2, the assumption

$$
H_{1}(z) \cdot N T F_{1}(z)-H_{2}(z) \cdot S T F_{2}(z)=0
$$

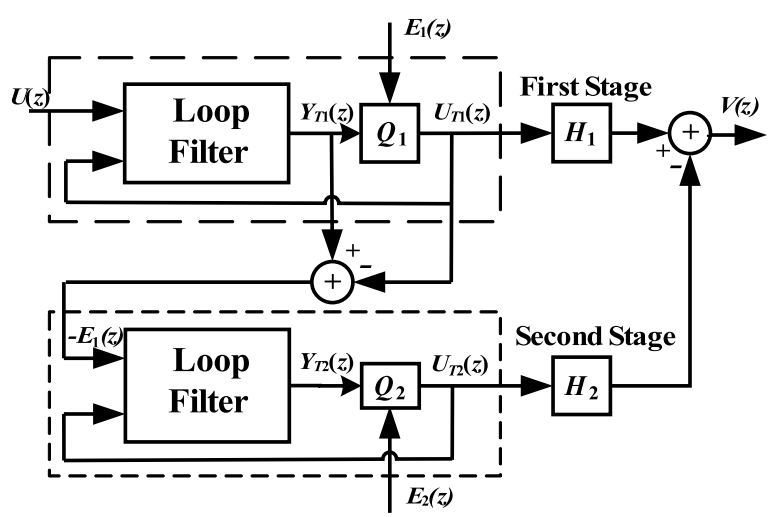

Fig. 4. A cascade structure of two-stage MASH modulators

must be absolutely true. For the 1-1 MASH $\Delta-\Sigma$ DPWM, the digital filter stages $H_{1}$ and $H_{2}$ are selected as

$$
\begin{gathered}
H_{1}(z)=\operatorname{STF}_{2}(z)=1 \\
H_{2}(z)=N T F_{1}(z)=1-z^{-1}
\end{gathered}
$$

However it still suffers from the idle tone existing in each stage and particularly the tone's overlapping between the two stages that introduce extra low-frequency interference without any attenuation in the baseband [17].

Figure 5 shows the transient simulation results by feeding 1025 (decimal) to a 11-bit DPWM (5-bit MOD) which indicates that the MOD2 still generates the idle tone compared to the MOD1. To avoid the idle tone, the MOD must be of high order and then have enough quantization levels. Unfortunately, more quantization levels that contain large offsets compared with the base digit will simultaneously lead to the instability problem that also deteriorates the output signal within a certain power stage filter. The undither MASH MOD, the same order as the MOD2, shows almost the same transient performance as MOD2. As shown in figure 6 , a serious transient variation occurs because the higher rate of output variations introduces extra digit-levels. So a dither module must be included here aiming to re-create the $\Delta-\Sigma$ sequence as well as suppress the idle tone effects.

According to [16], the input to the latter stage must be scaled to fit within the stable input range. So the bit extension module should be added here for the ease of calculation of the latter stage.

To avoid the large offset of digit, bit distribution through each stage is another important issue for designers. All referred bit distribution proposals belong to multi-bit quantizers. It is inherently more stable, and the no-overload range of the quantizer is increased, meanwhile it may have very high ENOB even at low OSR [16]. So two multi-bit quantizers are adopted in our design. Figure 7 shows the power spectral density (PSD) for $2+4$ (2MSB at the output of the first stage and $4 \mathrm{MSB}$ at the output of the second stage, similarly hereafter), $3+3$, $4+2$. Here the switching frequency is $1 \mathrm{MHz}$, meanwhile the 


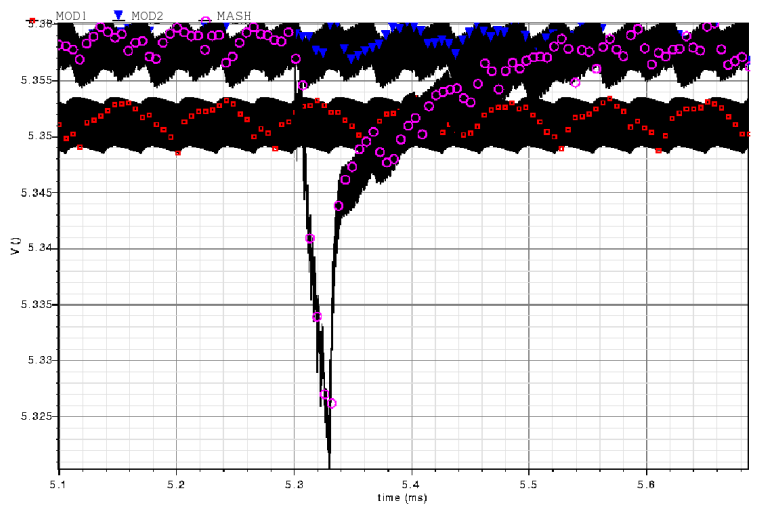

Fig. 5. Idle tone interferences within the simulation of MOD1, MOD2 and undither MASH MOD by feeding a constant DPWM input signal (1025 of 11-bit DPWM)
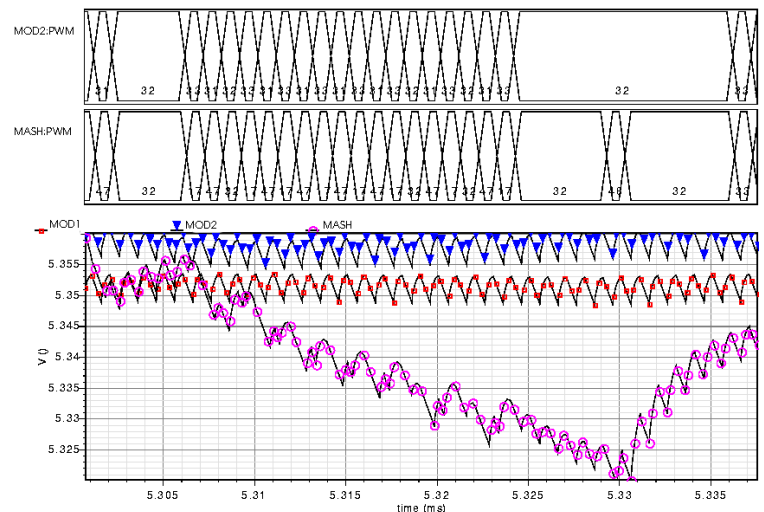

Fig. 6. Enlarged waveform of the transient variation point

core frequency is around $18.6 \mathrm{KHz}$ in our design. According to figure 7, 2+4 modulation structure is a better choice.

According to figure 7 , some noise signals below corner frequency still exist due to the idle tone effects, although they are significantly attenuated. Therefore dither method is proposed here to further suppress the idle tone, also known as improving the noise-shaping effect. Inspired by [24], a $(n-1)$ bit linear feedback shift register (LFSR) is added to generate the dither signal to the second stage as shown in figure 8 . The dither module is placed just before the quantizer because the dithering signal can behave as the same manner as the quantization noise and then permit a large amount of dithering. The Fibonacci LFSR is adopted here as show in figure 9. Here "01101101101"is set as the initial code in register array. The corresponding feedback polynomial is

$$
x^{11}+x^{9}+x^{7}+x^{5}+1
$$

where ' 1 ' corresponds to the input of the first bit. Finally the overall $m=6$ bits are sent to a 6-bit core DPWM which is implemented by the counter-comparator DPWM that brings down the maximum frequency from $2^{11} \cdot f_{s w}$ to $2^{6} \cdot f_{s w}$.

So the overall output signal is given by

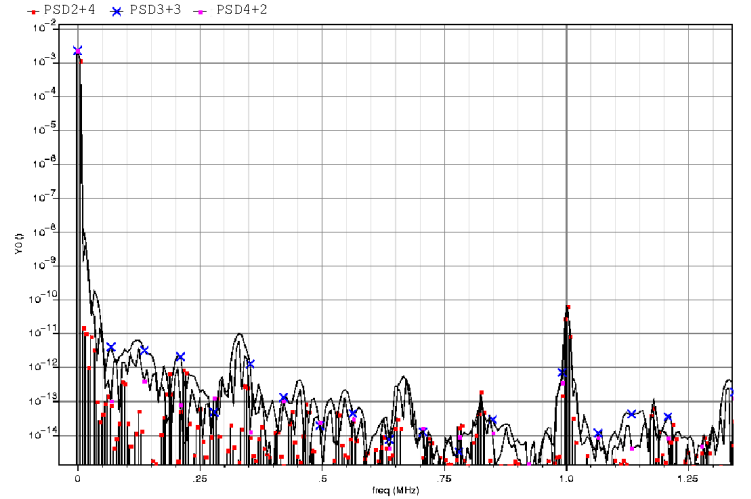

Fig. 7. Noise-shaping comparison between three types 1-1 MASH $\Delta-\Sigma$ modulations

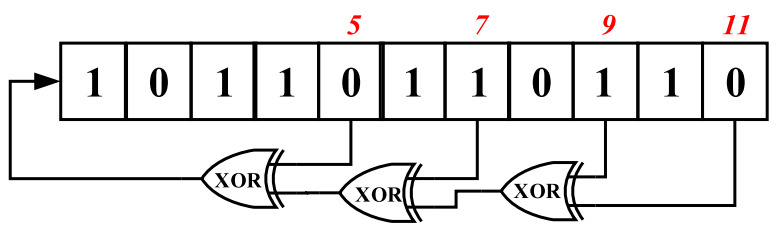

Fig. 9. Proposed dither 1-1 MASH $\Delta-\Sigma$ DPWM

$$
\begin{array}{r}
V(z)=U(z)+\left(1-z^{-1}\right)^{2} E_{2}(z) \\
+\frac{1}{n-1}\left(1-z^{-1}\right)^{2} D(z)
\end{array}
$$

where $D(z)$ is the dither signal, $E_{2}(z)$ is the truncation error of the second stage. As above-mentioned, we set $m_{1}=2$ and $m_{2}=4$. The output spectrum indicates the idle tone attenuation effects as shown in figure 10 .

\section{EXPERIMENTAL RESULTS}

To verify the function and timing issues of the proposed MASH $\Delta-\Sigma$ DPWM with SMC, a low voltage boost converter is connected to a Virtex-II Pro XC2VP30 FPGA board as

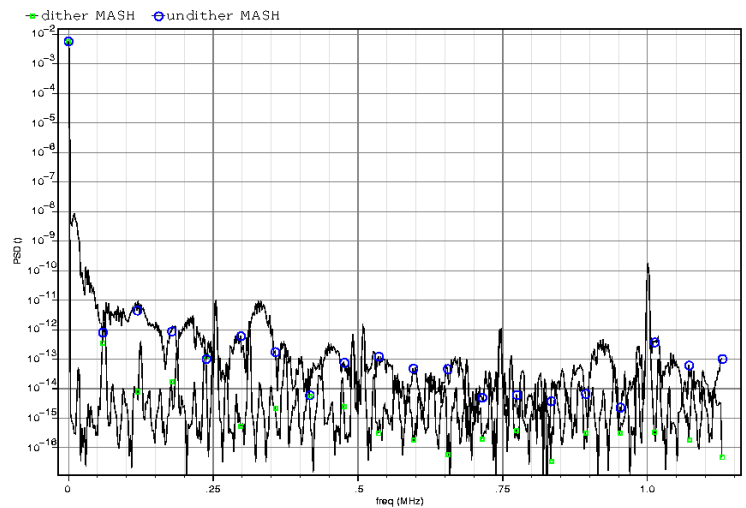

Fig. 10. Idle tone attenuation comparison between dither and undither 1-1 MASH modulator 


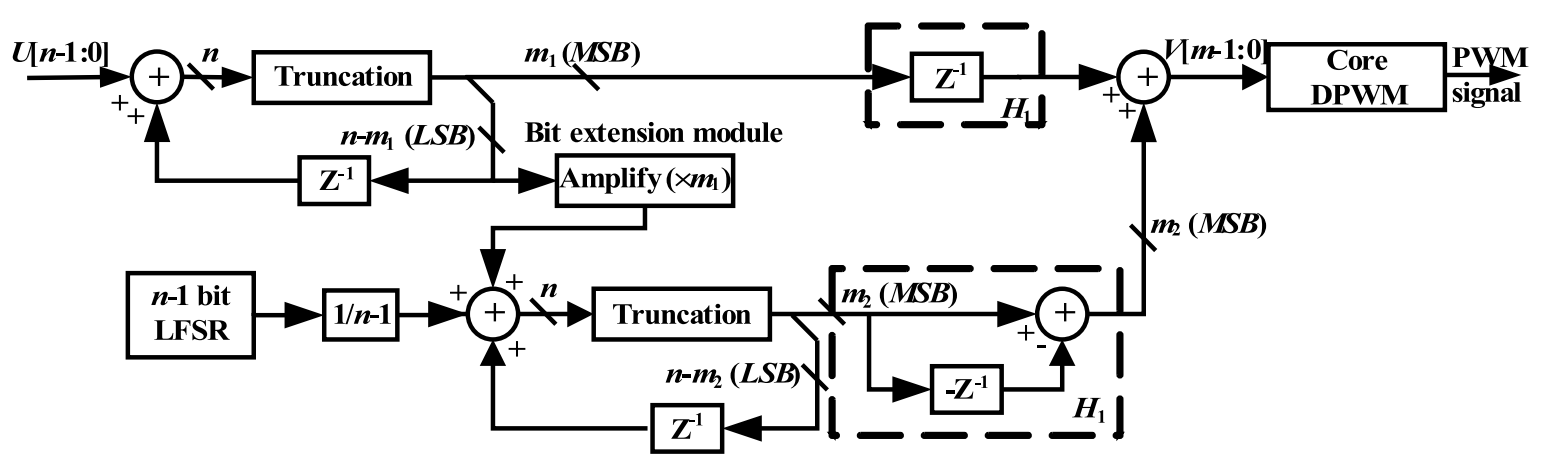

Fig. 8. Proposed dither 1-1 MASH $\Delta-\Sigma$ DPWM

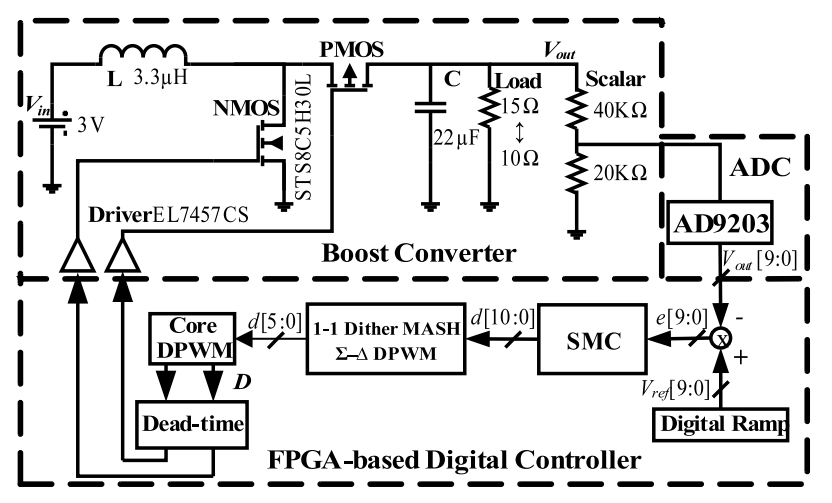

Fig. 11. Circuit configuration of the FPGA-based digital controller for boost converter

TABLE I

PARAMETERS OF THE DIGITALLY CONTROLLED BOOST CONVERTER

\begin{tabular}{ccc}
\hline \hline$R$ & Nominal load & $15 \Omega$ \\
$L$ & Inductor & $3.3 \mu \mathrm{H}$ \\
$C$ & Capacitor & $22 \mu \mathrm{F}$ \\
$V_{\text {in }}$ & Input voltage & $3.0 \mathrm{~V}$ \\
$V_{\text {out }}$ & Output voltage & $5.0 \mathrm{~V}$ \\
$f_{s w}$ & Switching frequency & $1 \mathrm{MHz}$ \\
$A D C$ & AD9203 & $10-\mathrm{bit}$ \\
$N_{\Sigma-\Delta}$ & MASH $\Sigma-\Delta$ resolution & $5-\mathrm{bit}$ \\
$N_{\text {Core_DPWM }}$ Core DPWM resolution & 6-bit \\
\hline \hline
\end{tabular}

shown in Figure 11. Subtracting the quantized output of the boost converter, the digital ramp module generates a ramp signal that assures a smooth start process. Dead-time module handles the output of the DPWM to avoid the shoot-through issue. Briefly speaking, it is necessary to preserve an actual safe interval between the two ON-states of the power MOSFETs. From the device utilization report, the total equivalent gate count for design is 42288 with 1776 additional JTAG gates for IOBs. Figure 12 is the experiment platform in which board 1 is the boost converter and board 2 is the ADC. Basic configuration parameters are shown in Table I.

Figure 13 shows the output voltage and the corresponding high-side PWM signal of the digital SMC controller in steadystate operation at $1 \mathrm{MHz}$. It exhibits sufficient stability in the output voltage. Figure 14 shows the transient output voltage

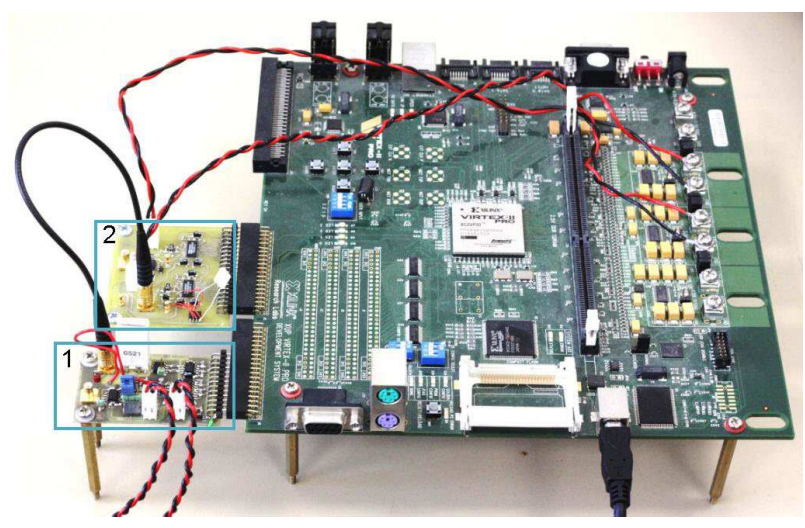

Fig. 12. The FPGA-based experiment platform for the proposed digital controller

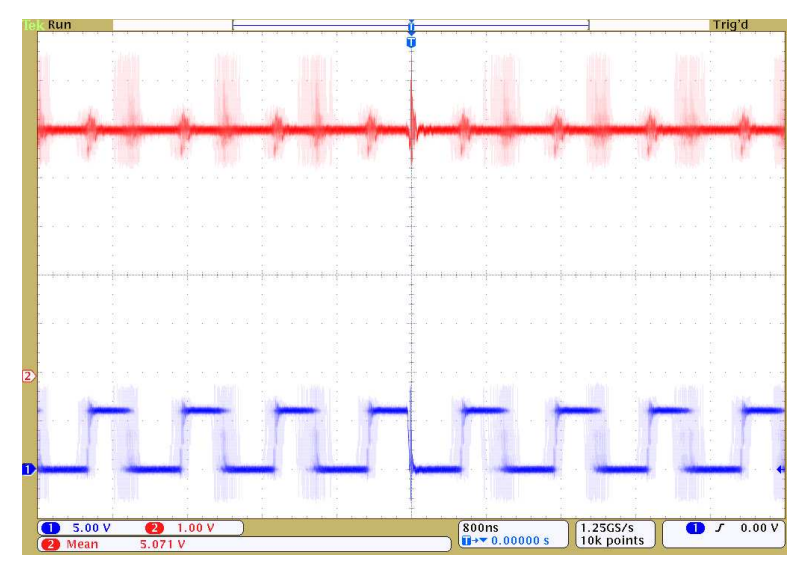

Fig. 13. High-side PWM and output voltage in steady state at $f_{s w}=1 \mathrm{MHz}$

response when load varies from $15 \Omega$ to $10 \Omega$ (using dither 1-1 MASH $\Delta-\Sigma$ DPWM). It indicates that the proposed SMC has satisfactory dynamic performances. These results prove that the dynamic response is very fast $(<25 \mu \mathrm{s})$ and the undershoot on the output voltage is also very small $(<70 \mathrm{mV})$ which is less than $1.4 \%$ of the output voltage $(5 \mathrm{~V})$. The performance of SMC is summarized in Table II compared with a PID controller. Nevertheless, this test executes a conservative load variation exclusively in order to guarantee to work in the 


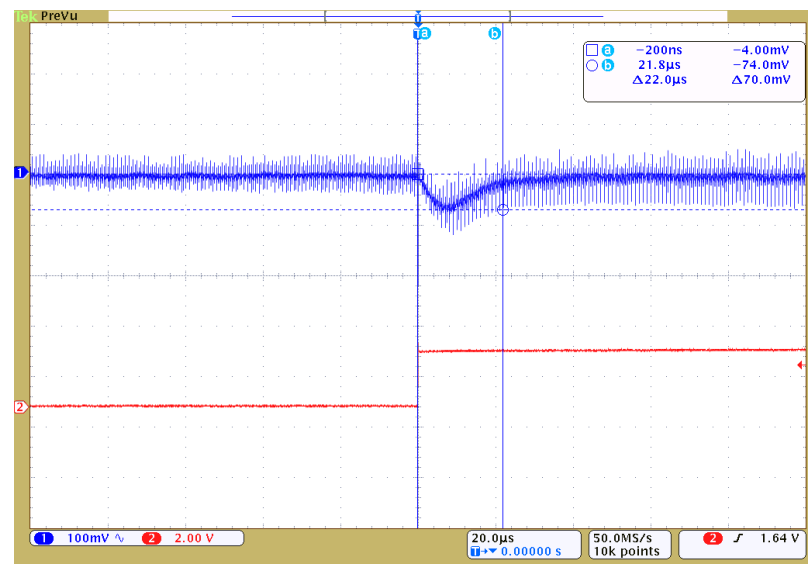

Fig. 14. Transient output voltage response when load goes from $15 \Omega$ to $10 \Omega$

TABLE II

PERFORMANCE SUMMARY

\begin{tabular}{cccc}
\hline \hline Load change & Performance index & SMC & PID Controller \\
\hline $15 \Omega$ & Recovery time & $25 \mu \mathrm{s}$ & $80 \mu \mathrm{s}$ \\
to & Undershoot & $70 \mathrm{mV}$ & $86 \mathrm{mV}$ \\
$10 \Omega$ & Output ripple & $30 \mathrm{mV}$ & $30 \mathrm{mV}$ \\
\hline $10 \Omega$ & Recovery time & $42 \mu \mathrm{s}$ & $102 \mu \mathrm{s}$ \\
to & Overshoot & $90 \mathrm{mV}$ & $92 \mathrm{mV}$ \\
$15 \Omega$ & Output ripple & $30 \mathrm{mV}$ & $30 \mathrm{mV}$ \\
\hline \hline
\end{tabular}

continuous current mode (CCM). In effect, a load variation from $45 \Omega$ to $10 \Omega$ is available in a serious condition test. Moreover as a further improvement, it is possible to add an observer to obtain load change information which helps to further ameliorate the control performance.

\section{CONCLUSION}

Cooperating with optimized SMC, a dither module is added to the 1-1 MASH $\Delta-\Sigma$ DPWM to restrain the idle tone effects. The FPGA-prototype is implemented in the Virtex-II Pro XC2VP30 platform serving a $1 \mathrm{MHz}$ boost converter. The experimental results are satisfactory. Other nonlinear controller algorithms are under consideration so as to make a comparison with the controller proposed in this paper. A further ASIC in a $0.35 \mu \mathrm{m}$ CMOS process is estimated to occupy $0.32 \mathrm{~mm}^{2}$ of silicon area on condition that the core utilization ratio is $75 \%$, and the current consumption is roughly $100 \mu \mathrm{A} / \mathrm{MHz}$. Therefore this approach anticipates a better performance and estimates to archive higher power efficiency in ASIC.

\section{REFERENCES}

[1] C. Edwards and S. Spurgeon, Sliding Mode Control: Theory and Applications. Taylor and Francis, London, 1998.

[2] P. Mattavelli, L. Rossetto, G. Spiazzi, and P. Tenti, "General-purpose sliding-mode controller for DC-DC converter applications," in Power Electronics Specialists Conference, 1993. PESC '93 Record., 24th Annual IEEE, June 1993, pp. $609-615$.

[3] B. Cardoso, A. Moreira, B. Menezes, and P. Cortizo, "Analysis of switching frequency reduction methods applied to sliding mode controlled DC-DC converters," in Applied Power Electronics Conference and Exposition, 1992. APEC '92. Conference Proceedings 1992., Seventh Annual, Feb 1992, pp. 403-410.
[4] P. Mattavelli, L. Rossetto, and G. Spiazzi, "Small-signal analysis of DC-DC converters with sliding mode control," Power Electronics, IEEE Transactions on, vol. 12, no. 1, pp. 96-102, Jan 1997.

[5] M. Castilla, L. de Vicuna, M. Lopez, O. Lopez, and J. Matas, "On the design of sliding mode control schemes for quantum resonant converters," Power Electronics, IEEE Transactions on, vol. 15, no. 6 , pp. 960-973, Nov 2000.

[6] S.-C. Tan, Y. Lai, and C. Tse, "General Design Issues of SlidingMode Controllers in DC-DC Converters," Industrial Electronics, IEEE Transactions on, vol. 55, no. 3, pp. 1160-1174, March 2008

[7] G. Chu, S.-C. Tan, C. Tse, and S. C. Wong, "General control for boost PFC converter from a sliding mode viewpoint," in Power Electronics Specialists Conference, 2008. PESC 2008. IEEE, June 2008, pp. 44524456.

[8] S.-C. Tan, Y. Lai, and C. Tse, "Indirect Sliding Mode Control of Power Converters Via Double Integral Sliding Surface," Power Electronics, IEEE Transactions on, vol. 23, no. 2, pp. 600-611, March 2008.

[9] S.-C. Tan, Y. Lai, C. Tse, and M. Cheung, "A fixed-frequency pulsewidth modulation based quasi-sliding-mode controller for buck converters," Power Electronics, IEEE Transactions on, vol. 20, no. 6, pp. $1379-$ 1392, 2005.

[10] H. Peng, D. Maksimovic, A. Prodic, and E. Alarcon, "Modeling of quantization effects in digitally controlled dc-dc converters," in Power Electronics Specialists Conference, 2004. PESC 04. 2004 IEEE 35th Annual, vol. 6, 2004, pp. 4312 - 4318 Vol.6.

[11] A. Peterchev and S. Sanders, "Quantization resolution and limit cycling in digitally controlled PWM converters," Power Electronics, IEEE Transactions on, vol. 18, no. 1, pp. 301-308, Jan 2003.

[12] B. Patella, A. Prodic, A. Zirger, and D. Maksimovic, "High-frequency digital pwm controller ic for dc-dc converters," Power Electronics, IEEE Transactions on, vol. 18, no. 1, pp. 438-446, Jan 2003

[13] T. Takayama and D. Maksimovic, "Digitally controlled $10 \mathrm{mhz}$ monolithic buck converter," in Computers in Power Electronics, 2006. COMPEL '06. IEEE Workshops on, 2006, pp. $154-158$.

[14] O. Trescases, G. Wei, and W. T. Ng, "A segmented digital pulse width modulator with self-calibration for low-power smps," Dec. 2005, pp. 367-370.

[15] J. Xiao, A. Peterchev, J. Zhang, and S. Sanders, "A 4- $\mu$ A quiescentcurrent dual-mode digitally controlled buck converter ic for cellular phone applications," Solid-State Circuits, IEEE Journal of, vol. 39 no. 12, pp. 2342-2348, Dec. 2004.

[16] R. Schreirar. and G. Temes, Delta-Sigma Data Converters. New York Wiley, 2005.

[17] Z. Lukic, N. Rahman, and A. Prodic, "Multibit $\Sigma-\Delta$ PWM Digital Controller IC for DC-DC Converters Operating at Switching Frequencies Beyond $10 \mathrm{MHz}$," Power Electronics, IEEE Transactions on, vol. 22 no. 5, pp. 1693-1707, Sept. 2007

[18] S. Guo, X. Lin-Shi, B. Allard, B. Li, Y. Gao, and Y. Ruan, "Highresolution digital PWM controller for high-frequency low-power SMPS," in Power Electronics and Applications, 2009. EPE '09. 13th European Conference on, 2009, pp. 1 -9.

[19] G. Chu, S.-C. Tan, C. Tse, and S. C. Wong, "General control for boost pfc converter from a sliding mode viewpoint," June 2008, pp. 4452 4456.

[20] F. Yorgancioglu and H. Kmrcgil, "Single-input fuzzy-like moving sliding surface approach to the sliding mode control," Elect Eng, vol. 90, pp. 199-207, 2008.

[21] J. Slotine and W. Li, Applied nonlinear control. Englewood Cliffs (New Jersey): Prentice Hall Inc., 1991.

[22] Z. Lukic, K. Wang, and P. Prodic, "High-frequency digital controller for DC-DC converters based on multi-bit $\Sigma-\Delta$ pulse-width modulation," in Applied Power Electronics Conference and Exposition, 2005. APEC 2005. Twentieth Annual IEEE, vol. 1, March 2005, pp. 35-40 Vol. 1.

[23] D. R.W.Ericksonand, Fundamentals of Power Electronics. Boston, MA Kluwer, 2000.

[24] V. Gonzalez-Diaz, M. Garcia-Andrade, G. Flores-Verdad, and F. Maloberti, "Efficient dithering in mash sigma-delta modulators for fractional frequency synthesizers," Circuits and Systems I: Regular Papers, IEEE Transactions on, vol. 57, no. 9, pp. $2394-2403,2010$. 